\title{
Interactions of quarkonium at low energies
}

\author{
H. Fujii a and D. Kharzeev ${ }^{\mathrm{b}}$ \\ anstitute of Physics, University of Tokyo at Komaba, Tokyo 153-8902, Japan. \\ ${ }^{\text {b} R I K E N ~ B N L ~ R e s e a r c h ~ C e n t e r, ~ B r o o k h a v e n ~ N a t i o n a l ~ L a b o r a t o r y, ~}$ \\ Upton, NY 11973, USA
}

We present two examples of the short-distance QCD calculations for the quarkonium $(\Phi)$ interactions; 1) $\pi \Phi$ elastic and $\pi \Phi \rightarrow \pi \Phi^{\prime}$ cross sections, and 2) the potential between two $\Phi$ 's. The former is relevant for the $\Phi$ suppression in heavy ion collisions; we find that the corresponding cross sections are very small. For the latter we derive the sum rule which relates the strength of the potential to the energy density of the QCD vacuum. The key starting point in both cases is that the leading operator in the operator product expansion (OPE) for the low-energy scattering amplitude is the trace of the QCD stress tensor whose matrix elements are unambiguously fixed by the low energy theorem. The resonances in the two-pion scalar channel are taken into account as a formfactor.

\section{INTRODUCTION}

The heavy quarkonium (for which we will use a generic notation $\Phi$ ) has a small size $\left(r \sim 1 /\left(\alpha_{s} m_{Q}\right)\right)$ and a large binding energy $\left(\epsilon \sim \alpha_{s}^{2} m_{Q}\right)$. When we consider an interaction with light hadrons $\left(h, h^{\prime}\right)$ at a low (compared to $\epsilon$ ) energy, the amplitude can be expanded in multipoles [1] 3]: $\mathcal{M}=\sum_{i} c_{i}\left\langle h^{\prime}\left|O_{i}(0)\right| h\right\rangle$, where $c_{i}$ are the Wilson coefficients (polarizabilities) which reflect the structure of the quarkonium. The matrix elements of the gauge-invariant local operators $O_{i}(x)$ over the light hadron state contain the information about the long distance part of the process; we assume that the factorization scale in this formula is $\epsilon$.

This approach has been applied to the evaluation of inclusive cross sections of heavy quarkonium dissociation in hadron gas in [4]. Here we evaluate exclusive cross sections of $\pi \Phi$ interactions. Previously these interactions were addressed in Refs. [5 7]; detailed description of our formalism and results can be found in Ref. [8].

The leading operator in OPE for the amplitude is $\frac{1}{2} g^{2} \mathbf{E}^{a 2}$, which describes the emission of two gluons in the color-singlet state. (The magnetic coupling is suppressed by the velocity $v \sim \alpha_{s} \ll 1$ for a heavy $\Phi$.) One can re-write this operator in the Lorentz covariant form, $\frac{1}{2} g^{2} \mathbf{E}^{a 2}=\frac{4 \pi^{2}}{9} \theta_{\mu}^{\mu}+\frac{1}{2} g^{2} \theta_{00}^{(G)}$. As will be discussed below, the scale anomaly implies that the matrix elements of the stress tensor, $\theta_{\mu}^{\mu}=-\left(9 g^{2} / 32 \pi^{2}\right)\left(G_{\mu \nu}^{a}\right)^{2}$ do not depend on the coupling $g^{2}$ and dominate over the matrix elements of the gluon tensor operator, $\theta_{00}^{(G)}$, which is manifestly suppressed by $g^{2}$. In other words, scale anomaly effectively eliminates the factor of $g^{2}$ in the amplitude! 


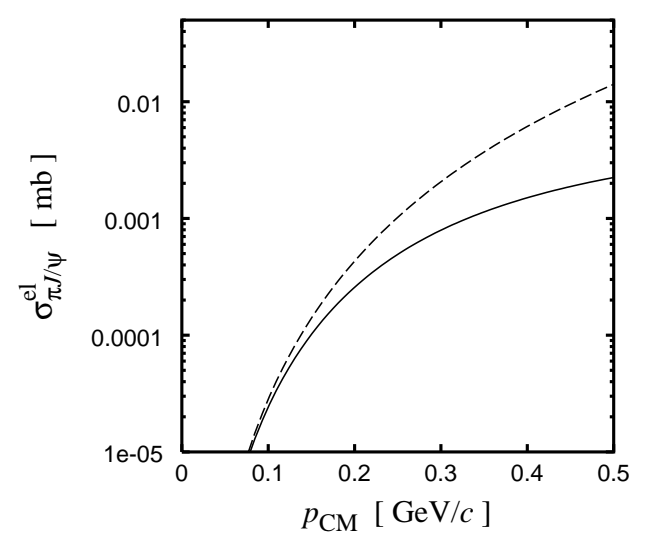

Figure 1. $\pi J / \psi$ elastic cross section as a function of the CM momentum; solid line is the complete result with the $\pi \pi$ formfactor $F$; dashed line shows the case without $F$.

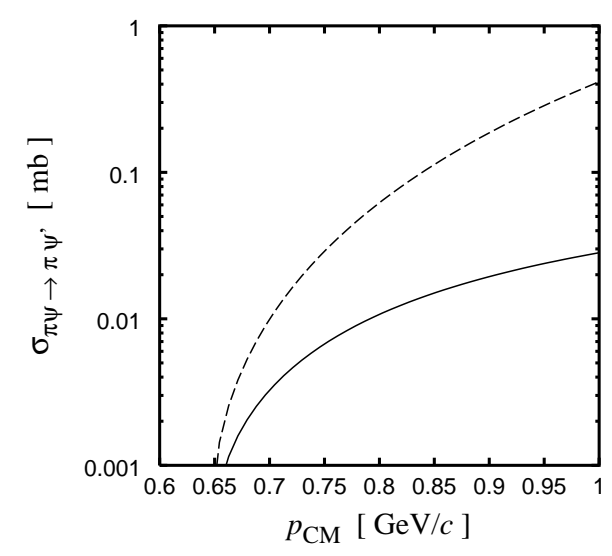

Figure 2. $\pi J / \psi \rightarrow \pi \psi^{\prime}$ transition cross section; for notations, see the caption to Fig. 1.

\section{2. $\pi \Phi$ INTERACTIONS}

Let us consider $\Phi$ interactions with pions, which are the dominant degrees of freedom at low energies/temperatures. Since $\theta_{\mu}^{\mu}$ is scale invariant, we can match it onto its expression in terms of the effective pion lagrangian, and find a beautiful relation [9] $\left\langle\pi^{+}\left(p^{\prime}\right)\left|\theta_{\mu}^{\mu}\right| \pi^{+}(p)\right\rangle=\left(p^{\prime}-p\right)^{2}=t$ valid in the chiral limit. In the leading order in OPE, the amplitude of the $\pi \Phi$ elastic scattering can be written as

$\mathcal{M}^{\Phi \pi}=\bar{d}_{2} \frac{a_{0}^{2}}{\epsilon_{0}} \frac{4 \pi^{2}}{9}\left\langle\pi^{+}\left(p^{\prime}\right)\left|\theta_{\mu}^{\mu}\right| \pi^{+}(p)\right\rangle=\bar{d}_{2} \frac{a_{0}^{2}}{\epsilon_{0}} \frac{4 \pi^{2}}{9} t F(t)$,

where, in the polarizability $\bar{d}_{2} a_{0}^{2} / \epsilon_{0}$, we have factored out the Bohr radius $a_{0}$ and the Rydberg energy $\epsilon_{0}$ of quarkonium, respectively; $F(t)$ is the pion formfactor in the scalarisoscalar channel, which takes account of the resonances in this channel.

The resulting elastic cross section of $\Phi$ with mass $M$

$\sigma^{\mathrm{el}}(s)=\frac{1}{4 \pi s} \frac{M^{2}}{4 p_{\mathrm{cm}}^{2}}\left(\bar{d}_{2} \frac{a_{0}^{2}}{\epsilon_{0}}\right)^{2}\left(\frac{4 \pi^{2}}{9}\right)^{2} \int_{0}^{4 p_{\mathrm{cm}}^{2}} d(-t) t^{2}|F(t)|^{2}$

appears to be very small (see Fig. 1) because of the factor $a_{0}^{4} / \epsilon_{0}^{2}$ stemming from the doubledipole nature of the interaction. Moreover, it is further suppressed by the dependence on the momentum transfer $t$ of the cross section dictated by the chiral symmetry.

In Fig. 1 we show the $\pi \Phi$ elastic cross section for the $J / \psi$ case; one can see that the cross section is very small. This result implies that the $J / \psi$ 's produced in heavy ion 
collisions interact with the surrounding $\pi$ gas very weakly. The $p_{T}$ broadening of the $\Phi$ 's by the interactions inside the pion gas therefore is negligible.

Next we consider the inelastic excitation process, $\pi \Phi \rightarrow \pi \Phi^{\prime}$. The energy transfer in this process is on the order of the binding energy, $\Delta=M^{\prime}-M=O\left(\epsilon_{0}\right)$, which may invalidate our starting assumption of the factorization. Fortunately the double-dipole form is intact, but non-local in time. We replace the gluon energy appearing in the energy denominator with the typical value, $\Delta$, as an approximation, to make the gluonic operator local:

$\mathcal{M}^{\pi \Phi \rightarrow \pi \Phi^{\prime}} \simeq \frac{1}{3 N_{c}}\left\langle\phi^{\prime}\left|r^{i} \frac{1}{H_{a}+\epsilon-\Delta} r^{i}\right| \phi\right\rangle\left\langle\pi\left|\frac{1}{2} g^{2} \mathbf{E}^{2}\right| \pi\right\rangle \equiv \bar{d}_{2}^{\prime} \frac{a_{0}^{2}}{\epsilon_{0}}\left\langle\pi\left|\frac{1}{2} g^{2} \mathbf{E}^{2}\right| \pi\right\rangle$,

where the $\phi\left(\phi^{\prime}\right)$ is the internal wave function of $\Phi\left(\Phi^{\prime}\right)$, and $H_{a}(r)$ is the effective hamiltonian describing the intermediate, $S U\left(N_{c}\right)$ color-adjoint quark-anti-quark state. With this amplitude we get the transition cross section shown in Fig. 2.

Our replacement of the gluon energy in the energy denominator is an approximation with an accuracy that cannot be evaluated a priori. We can, however, apply our formula to the decay process $\psi^{\prime} \rightarrow J / \psi \pi \pi$ and compare our results, $\Gamma^{\pi \pi}=260$ (70) keV obtained with (without) taking account of the formfactor $F(s)$, with the experimentally measured value of $135 \pm 20 \mathrm{keV}$. From this comparison we conclude that our calculations are probably valid up to a factor of 2 .

Our results support the idea 10,4 that the interactions of heavy quarkonia in the hadron gas and in the quark-gluon plasma are very different.

\section{ONIUM-ONIUM SCATTERING AT LOW ENERGY}

Low-energy onium-onium scattering is a very interesting subject, which was addressed by several authors [11,21,12]. One may regard this problem as a first step toward the understanding of nuclear force from QCD. Here we derive a sum rule for this interaction 8.

After the multipole expansion for the interactions of both $\Phi$ 's with the gluon field is performed, the potential between them can be expressed through the $\theta_{\mu}^{\mu}$ correlator:

$$
\begin{aligned}
V(R)=-\mathcal{M}^{\Phi \Phi}(R) & =-i \int_{\infty}^{\infty} d t\left(\bar{d}_{2} \frac{a_{0}^{2}}{\epsilon_{0}}\right)^{2}\left(\frac{4 \pi^{2}}{b}\right)^{2}\left\langle 0\left|\mathrm{~T} \theta_{\mu}^{\mu}(x) \theta_{\nu}^{\nu}(0)\right| 0\right\rangle \\
& =-\left(\bar{d}_{2} \frac{a_{0}^{2}}{\epsilon_{0}}\right)^{2}\left(\frac{4 \pi^{2}}{b}\right)^{2} \int_{0}^{\infty} d \sigma^{2} \rho_{\theta}\left(\sigma^{2}\right) \frac{1}{4 \pi R} e^{-\sigma R}
\end{aligned}
$$

where we have introduced the spectral function for the $\theta_{\mu}^{\mu}$ correlator as $\left\langle 0\left|\mathrm{~T} \theta_{\mu}^{\mu}(x) \theta_{\nu}^{\nu}(0)\right| 0\right\rangle=$ $\int d \sigma^{2} \rho_{\theta}\left(\sigma^{2}\right) \Delta_{F}\left(x ; \sigma^{2}\right)$ with the coordinate-space scalar Feynman propagator, $\Delta_{F} ; b=$ $\left(11 N_{c}-2 N_{f}\right) / 3=9$.

An important theorem 13 for the $\theta_{\mu}^{\mu}$ correlator relates it to the non-perturbative energy density of the QCD vacuum:

$i \int d^{4} x e^{i q x}\left\langle 0\left|\mathrm{~T} \theta_{\mu}^{\mu}(x) \theta_{\nu}^{\nu}(0)\right| 0\right\rangle=\int d \sigma^{2} \frac{\rho_{\theta}\left(\sigma^{2}\right)}{\sigma^{2}-q^{2}-i \epsilon} \stackrel{q \rightarrow 0}{\longrightarrow}-4\left\langle 0\left|\theta_{\mu}^{\mu}(0)\right| 0\right\rangle=-16 \epsilon_{\mathrm{vac}}$. 
Since the vacuum energy density is divergent in perturbation theory, we define the r.h.s. by subtracting the perturbative part. This relates the integral of the spectral density to $\epsilon_{\mathrm{vac}}$ as $\int\left(d \sigma^{2} / \sigma^{2}\right)\left(\rho_{\theta}^{\mathrm{phys}}\left(\sigma^{2}\right)-\rho_{\theta}^{\mathrm{pt}}\left(\sigma^{2}\right)\right)=-16 \epsilon_{\mathrm{vac}}$. In the heavy quark limit, this relation leads to the following sum rule for the potential

$$
\int_{r}^{\infty} d^{3} \mathbf{R}\left(V_{\theta}(R)-V_{\theta}^{\mathrm{pt}}(R)\right)=-\left(\bar{d}_{2} \frac{a_{0}^{2}}{\epsilon_{0}}\right)^{2}\left(\frac{4 \pi^{2}}{b}\right)^{2} 16\left|\epsilon_{\mathrm{vac}}\right|
$$

where $r$ is the size of the onium. This sum rule relates the overall strength of the interaction between the dipoles to the energy density of the QCD vacuum.

The short- and long-distance limits of the potential are analyzed in Ref. [8]. In particular, it appears that the long-distance limit of the potential has an interesting $R$ dependence, $\sim R^{-5 / 2} \exp \left(-2 \mu_{\pi} R\right)$ ( $\mu_{\pi}$ is the pion mass), with a non-trivial dependence on the numbers of colors $N_{c}$ and flavors $N_{f}, \sim\left(N_{f}^{2}-1\right)^{2} /\left(11 N_{c}-2 N_{f}\right)^{2}$.

\section{REFERENCES}

1. K. Gottfried, Phys. Rev. Lett. 40, 598 (1978);

M.B. Voloshin, Nucl. Phys. B154, 365 (1979).

2. M.E. Peskin, Nucl. Phys. B156, 365 (1979);

G. Bhanot and M.E. Peskin, Nucl. Phys. B156, 391 (1979).

3. A. Kaidalov, in QCD and High-Energy Interactions, J. Trân Thanh Vân (Ed.) Editions Fronti'eres, 1993.

4. D. Kharzeev and H. Satz, Phys. Lett. B334, 155 (1994).

5. D. Kharzeev, in Selected Topics in Nonperturbative QCD, A. Di Giacomo and D. Diakonov (Eds.), IOS, 1996; nucl-th/9601029.

6. H. Sorge, E. Shuryak and I. Zahed, Phys. Rev. Lett. 79, 2775 (1997).

7. J.-W. Chen and M.J. Savage, Phys. Rev. D 57, 2837 (1998).

8. H. Fujii and D. Kharzeev, hep-ph/9903495.

9. M.B. Voloshin and V.I. Zakharov, Phys. Rev. Lett. 45, 688 (1980);

V.A. Novikov and M.A. Shifman, Z. Phys. C8, 43 (1981).

10. T. Matsui and H. Satz, Phys. Lett. B178, 416 (1986).

11. T. Appelquist, and W. Fischler, Phys. Lett. 77B, 405 (1978).

12. S.J. Brodsky and G.A. Miller, Phys. Lett. B412, 125 (1997).

13. V.A. Novikov, M.A. Shifman, A.I. Vainstein and V.I. Zakharov, Nucl. Phys. B165, 67 (1980). 